\title{
INVESTIGATING THE POSSIBILITY OF PREPARING SMALL SCALE SOIL MOISTURE MAP FROM COUPLED SENTINEL-1 AND SENTINEL-2 DATA
}

\author{
R. Attarzadeh ${ }^{1, *}, \mathrm{~J}$. Amini $^{2}$ \\ ${ }^{1}$ Dept. of Surveying and Geomatics Engineering, Maybod Branch, Islamic Azad University, Maybod, Iran - \\ r.attarzadeh@outlook.com \\ ${ }^{2}$ School of Surveying and Geospatial Engineering, College of Engineering, University of Tehran, Tehran, Iran - \\ jamini@ut.ac.ir
}

Commission VI, WG VI/4

KEY WORDS: Soil Moisture Map, Object-Based Image Analysis, Multi-resolution Segmentation, Sentinel-1, Sentinel-2, SMAP

\begin{abstract}
:
With the failure of the radar instrument on NASA's Soil Moisture Active Passive (SMAP) satellite, the Sentinel-1 sensor has been considered as an alternative for replacing the SMAP radar data and restoring the combined radar and radiometer SMC product. A challenging subject to this purpose is the immense discrepancy between the spatial resolution of planned SMAP radar instrument (3 $\mathrm{km})$ and Sentinel-1 data $(10 \mathrm{~m})$. In this paper, we investigate the possibility of preparing small scale soil moisture map and its quality from the synergy of Sentinel-1 and Sentinel-2 data using object-based image analysis (OBIA). To reach this goal, the most related features with soil moisture variable extracted from Sentinel-1 and Sentinel-2 data have been used as input layers to multi-resolution segmentation (MRS) algorithm to create image objects. Then the support vector regression (SVR) estimator has been used to calculate the soil moisture value of image objects. Initial evaluations demonstrate that produced soil moisture map obtained acceptable accuracy. In addition, the flexibility of the final product improves on the scale of the soil moisture map regarding the shape and size of image objects. It is also possible to combine this soil moisture product with a Level-3 SMAP SSM product to exploit the advantages of both products. This combination would lead to a small scale soil moisture map with enhanced accuracy and flexible scale.
\end{abstract}

\section{INTRODUCTION}

Soil moisture retrieval using remote sensing data has given rise to a considerable research interest over the past decades. Active microwave remote sensing has already revealed its potential in soil moisture retrieval with high spatial resolution imageries and independent of weather conditions. This capacity is due to the fact that microwave signals are influenced by dielectric properties (and thus the water content) of the soils (Wang, 1980). In recent years, there has been growing interest in multisensor fusion for retrieving soil moisture. Synthetic aperture radar (SAR) and optic data fusion are one of the most widely used approaches (Baghdadi et al., 2015; Hajj et al., 2017; Prakash, Singh, \& Pathak, 2012). The integration of data acquired by optical sensors and SAR data may provide useful information for reducing ambiguity due to the presence of vegetation. Another possible approach is the integration of active and passive microwave data that could help to retrieve soil moisture at a higher accuracy (Kolassa, Gentine, Prigent, Aires, \& Alemohammad, 2017; Kolassa, Reichle, \& Draper, 2017). The SMAP mission has been designed based on this assumption. In another perspective, since active and passive sensors provide an SMC map at large and low scales, respectively, previous works have focused on down and upscaling to retrieve soil moisture at the intermediate scale. This results from the fact that many projects require an SMC map with a retrieval scale that is different from that which is provided with the operating sensors (Attarzadeh \& Amini, 2019). In this paper, we developed a robust approach for producing small scale soil moisture content (SMC) map from the synergy of Sentinel-1 and Sentinel-2 data. Considering the
SMAP radar failure and focus of NASA on Sentinel-1 as the replacement for SMAP radar, this approach also can be considered as an alternative approach for combining Sentinel-1, Sentinel-2 and SMAP data to produce the related product.

\section{METHODOLOGY}

The proposed method has been applied to the Uasin Gishu County in Kenya with dominant vegetation cover (Fig. 1). The Sentinel-1 data have been acquired simultaneously with field measurements. The Sentinel-2 data have been obtained with a minimum difference with radar data acquisition time and taking into account the cloud coverage. Due to the limitation of radar data for retrieving soil moisture in the vegetated areas results from Sentinel-1 sensor operating polarization and frequency (single VV polarization in $\mathrm{C}$ band), the Sentinel-2 data have also been used in this study. Then, 38 features, including nine radar feature and 29 optical features have been extracted. The most related features with soil moisture parameter have been selected using Random Forest-Recursive Feature Elimination method and considering the field measurements. On account of the small training database (164 samples), the support vector regression (SVR) technique has been used to retrieve soil moisture values in pixel level with 4.94-vol. \% RMSE estimation accuracy. At the next phase, the selected features have been used as input layers to the segmentation process. Multi-resolution segmentation algorithm with appropriate scale parameter has been used to create image objects (Fig. 2 and 3). Afterwards, the extracted pixel-based SVR estimator has been exploited to retrieve soil moisture values of produced image objects (Attarzadeh, Amini, Notarnicola, \& Greifeneder, 2018).

\footnotetext{
* Corresponding author
} 
Table 1 details the statistics of image objects in the generated SMC map and Fig. 4 and 5 demonstrate the produced soil moisture map with the proposed approach.

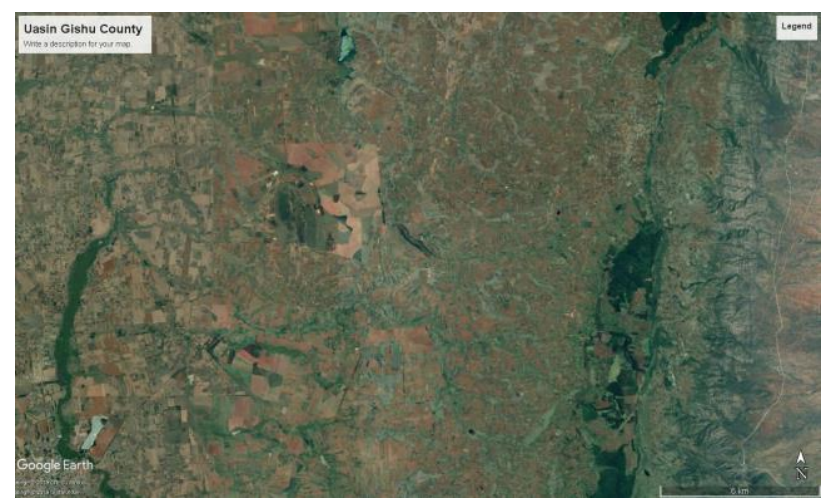

Figure 1. Google earth view of the study area (Uasin Gishu County, Kenya)

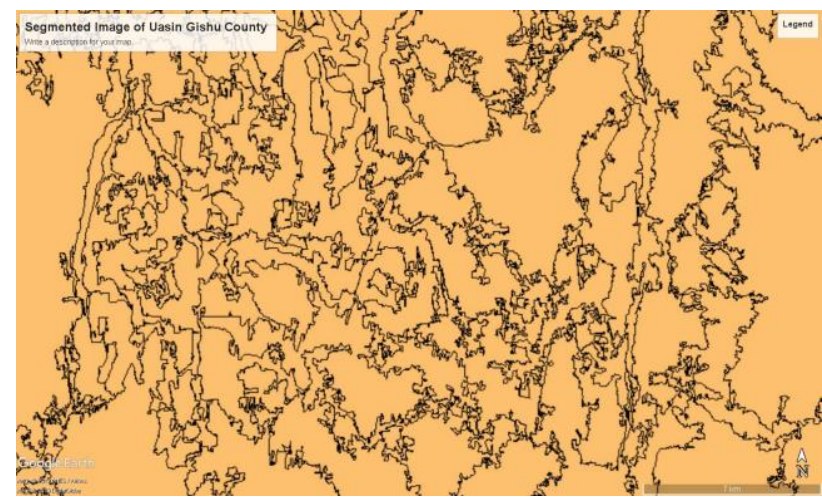

Figure 2. Segmented image of the study area with scale parameter 800 , shape 0.1 and compactness 0.5

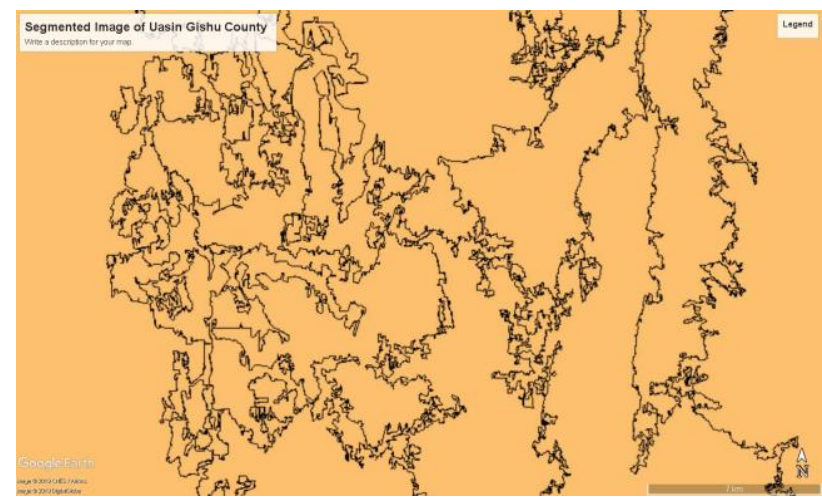

Figure 3. Segmented image of the study area with scale parameter 1600, shape 0.1 and compactness 0.5

\begin{tabular}{|c|c|c|c|c|c|c|}
\hline Scale Parameter & $\begin{array}{l}\text { No. of } \\
\text { image } \\
\text { objects }\end{array}$ & $\begin{array}{l}\text { No. of } \\
\text { pixels } \\
\text { Min }\end{array}$ & $\begin{array}{c}\text { No. of } \\
\text { pixels } \\
\text { Max }\end{array}$ & $\begin{array}{l}\text { No. of pixels } \\
\text { Average }\end{array}$ & Area (ha) & Avg. Size $(\mathrm{m})$ \\
\hline SP-100 & 5111 & 201 & 10006 & 848 & 8.48 & 291 \\
\hline SP-200 & 1129 & 29 & 29678 & 3842 & 38.42 & 620 \\
\hline SP-400 & 265 & 184 & 112874 & 16371 & 163.71 & 1280 \\
\hline SP-800 & 51 & 2258 & 351711 & 85066 & 850.66 & 2917 \\
\hline SP-1600 & 11 & 16999 & 853623 & 394400 & 3944.00 & 6280 \\
\hline
\end{tabular}

Table 1. Detailed statistics of the image objects for each scale parameter (Column 6: The average area of the image objects in hectare, Column 7: The average size of image objects for corresponding SP)

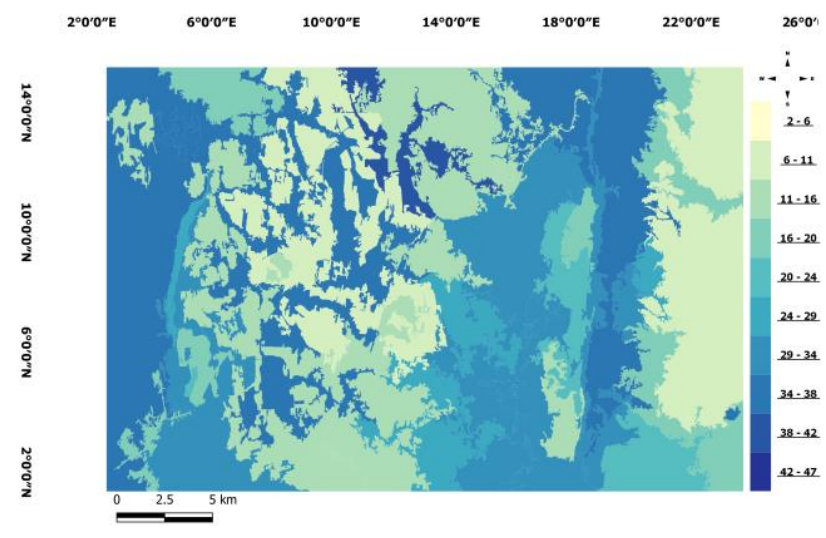

Figure 4. Object-based soil moisture map of the study area with scale parameter 800 and average objects area of $9 \mathrm{~km}^{2}$

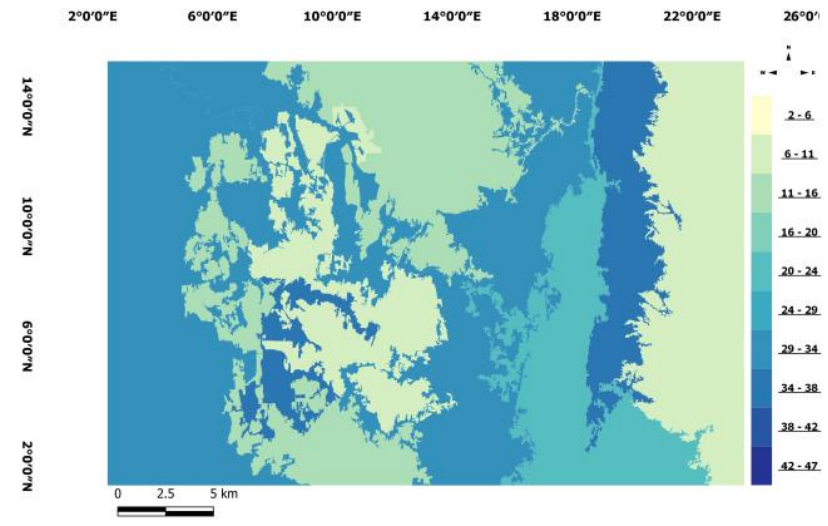

Figure 5. Object-based soil moisture map of the study area with scale parameter 1600 and average objects area of $40 \mathrm{~km}^{2}$

\section{RESULTS AND DISCUSSION}

Following the training step, the soil moisture retrieval process was performed by using the pixel-based estimator model. Attarzadeh et al. (2018) showed that the obtained estimation accuracy using the extracted SVR model was $4.94 \%$ (Vol.) and 0.89 in terms of the root-mean-square error (RMSE) and $\mathrm{R}^{2}$. As shown in Fig. 4, Fig. 5 and Table 1, the proposed method has the ability to produce small scale soil moisture map with approximate average object area of 9 and 40 square kilometres with corresponding 800 and 1600 scale parameter. This method also has this potential to produce soil moisture map with smaller image objects and consequently larger scale (Table 1). Since the default data for producing such small scale SMC map are passive data like SMAP, the proposed approach enable us to exploit the potential of free active and optic Sentinel data for preparing small scale SMC maps in object level and with the flexibility that it is not possible through rigid shape and size of pixels in SMAP SSM products. It should be noted that the OBIA procedure permits us to extract image objects with various sizes in an operational project so that fields of any magnitude can be identified and retrieved. This viewpoint will enable us to have a degree of flexibility on the retrieval scale. This approach seems to meet practical application requirements better. It is also possible to combine produced maps with SMAP products with a smaller spatial resolution to exploits the advantages of both products. 


\section{CONCLUSION}

This paper has investigated the possibility of producing small scale maps from coupled Sentinel-1 and Sentinel-2 data. The results of this study indicate that there is the potential to create such an SMC map using object-based image analysis with more flexibility on image objects size and shape. The most important limitation of the proposed approach lies in using a pixel-based SVR estimator to retrieve soil moisture values of image objects. Exploiting the pixel-based SVR estimator is a significant source of the unreliability of the SMC map's estimation accuracy. To further our research and to tackle the dependency of the estimation accuracy of the produced SMC map, we plan to combine this product with the SMAP product.

\section{ACKNOWLEDGEMENTS}

The authors would like to thank Claudia Notarnicola, Felix Greifeneder and also the Institute for Earth Observation of Eurac Research (Italy) for the support of this paper.

\section{REFERENCES}

Attarzadeh, R., Amini, J., 2019. Towards an object-based multiscale soil moisture product using coupled Sentinel-1 and Sentinel-2 data. Remote Sensing Letters, 10(7), 619-628. https://doi.org/10.1080/2150704X.2019.1590872

Attarzadeh, R., Amini, J., Notarnicola, C., Greifeneder, F., 2018. Synergetic Use of Sentinel-1 and Sentinel-2 Data for Soil Moisture Mapping at Plot Scale. Remote Sensing, 10(8), 1285. https://doi.org/10.3390/rs10081285

Baghdadi, N. N., Hajj, M. El, Zribi, M., Fayad, I., 2015. Coupling SAR C-Band and Optical Data for Soil Moisture and Leaf Area Index Retrieval Over Irrigated Grasslands. IEEE Journal of Selected Topics in Applied Earth Observations and Remote Sensing, 9(3), 1-15. https://doi.org/10.1109/JSTARS.2015.2464698

Hajj, M. El, Baghdadi, N., Zribi, M., Bazzi, H., 2017. Synergic use of Sentinel-1 and Sentinel-2 images for operational soil moisture mapping at high spatial resolution over agricultural areas. Remote Sensing, 9(12), 1292. https://doi.org/10.3390/rs9121292

Kolassa, J., Gentine, P., Prigent, C., Aires, F., Alemohammad, S. H., 2017. Soil moisture retrieval from AMSR-E and ASCAT microwave observation synergy. Part 2: Product evaluation. Remote Sensing of Environment, 195, 202217. https://doi.org/10.1016/J.RSE.2017.04.020

Kolassa, J., Reichle, R. H., Draper, C. S., 2017. Merging active and passive microwave observations in soil moisture data assimilation. Remote Sensing of Environment, 191, 117130. https://doi.org/10.1016/J.RSE.2017.01.015

Prakash, R., Singh, D., Pathak, N. P., 2012. A Fusion Approach to Retrieve Soil Moisture With SAR and Optical Data. Selected Topics in Applied Earth Observations and Remote Sensing, IEEE Journal Of. https://doi.org/10.1109/JSTARS.2011.2169236

Wang, J. R., 1980. The dielectric properties of soil-water mixtures at microwave frequencies. Radio Science, 15(5), 977-985.

https://doi.org/10.1029/RS015i005p00977 